\title{
Vibrio ichthyoenteri sp. nov., a Pathogen of Japanese Flounder (Paralichthys olivaceus) Larvae
}

\author{
KATSUYA ISHIMARU, ${ }^{1}$ MASAYO AKAGAWA-MATSUSHITA, ${ }^{2}$ AND KIYOKUNI MUROGA ${ }^{1 *}$ \\ Faculty of Applied Biological Science, Hiroshima University, Higashihiroshima-shi $739,{ }^{1}$ and Department of Chemistry, \\ University of Occupational and Environmental Health, Yahatanishi-ku, Kitakyushu-shi 807, Japan
}

\begin{abstract}
Seven similar strains which were pathogens of Japanese flounder (Paralichthys olivaceus) larvae with opaque intestines had characteristics of the genus Vibrio. These strains were divided into two genomic species (species 1 containing six strains, and species 2 containing one strain) on the basis of the results of DNA-DNA hybridization experiments in which the membrane filter method was used, and these two species could be differentiated from each other by the following characteristics: acid production from D-galactose and utilization of D-glucuronate and $\beta$-hydroxybutyrate. Strain F-2, the type strain of species 1, exhibited levels of DNA relatedness with 29 previously described Vibrio species of 5 to $18 \%$. The flounder isolates belonging to species 1 were also differentiated from the previously described Vibrio species phenotypically by the following characteristics: they were nitrate reduction positive; each cell had a single polar flagellum; they did not produce arginine dihydrolase, chitinase, gelatinase, and lipase; they did not utilize D-cellobiose and citrate; and they did not grow at $35^{\circ} \mathrm{C}$. The $\mathrm{G}+\mathrm{C}$ contents of the DNAs of four species 1 strains were 43 to $44 \mathrm{~mol} \%$. The name Vibrio ichthyoenteri sp. nov. is proposed for genomic species 1 . The type strain of $V$. ichthyoenteri is strain F-2 (= IFO 15847). Species 2 was also considered a new genomic species, but a species name is not proposed in this paper because only one strain is available and the phenotypic variability of the species is not known.
\end{abstract}

Since 1971, a bacterial disease characterized by opaque intestines or intestinal necrosis and high rates of mortality has occurred often in larval Japanese flounder (Paralichthys olivaceus) reared at marine hatcheries in Japan (13). O. Murata (12) isolated the causative agent of the disease, a vibrio, from affected flounder larvae between 1971 and 1973 in Wakayama Prefecture, but the strains isolated by Murata have been lost. Between 1986 and 1988 we isolated the causative agent of the disease mainly in Hiroshima Prefecture. On the basis of its morphological, biochemical, physiological, pathological, and serological characteristics, this flounder pathogen, which was tentatively designated Vibrio species INFL (intestinal necrosis of flounder larvae), was thought to be a new species (11). The disease caused by Vibrio species INFL is a very unusual fish disease because a local (intestinal) infection develops only in larval fish (14). In this study we determined the taxonomic position of this flounder pathogen and propose the name Vibrio ichthyoenteri sp. nov. for it.

\section{MATERIALS AND METHODS}

Bacterial strains. The strains which we used are listed in Table 1. The seven strains belonging to the INFL group were isolated from the guts of diseased Japanese flounder larvae reared in prefectures in western Japan. These strains were isolated and subcultured on ZoBell $2216 \mathrm{e}$ agar plates (1) at $25^{\circ} \mathrm{C}$ and were maintained in semisolid ZoBell $2216 \mathrm{e}$ medium containing $0.5 \%$ agar at $20^{\circ} \mathrm{C}$; they were subcultured every 6 months. Aged and filtered seawater obtained from the Inland Sea of Japan was used to prepare ZoBell 2216e medium. The pathogenicity of three strains (strains F-1, F-2 $2^{\mathrm{T}}[\mathrm{T}=$ type strain], and FH-8804) for flounder larvae was confirmed experimentally (11).

In the DNA hybridization tests performed in this study we used the type strains of all species except Vibrio cholerae. It has been shown previously by DNA-DNA hybridization that the strain of $V$. cholerae used in this study (strain HUFP 5004 $[=$ NIH 35A3]) is closely related to the type strain of this species (strain ATCC 14035) (level of similarity, 98\%) (3).

Phenotypic characterization. Arginine dihydrolase activity (Thornley method) was determined as described by West and Colwell (19). Other phenotypic char-

* Corresponding author. Mailing address: Faculty of Applied Biological Science, Hiroshima University, 1-4-4 Kagamiyama, Higashihiroshima-shi, Hiroshima Prefecture, Japan 739. Phone: 0824-24-7977. Fax: 0824-22-7059. acteristics of the seven strains were determined as described previously (9). The $\mathrm{NaCl}$ concentration was adjusted to $1.5 \%(\mathrm{wt} / \mathrm{vol})$ in all of the test media except those containing seawater or those that were used to determine the range of $\mathrm{NaCl}$ concentrations for growth.

Genetic characterization. The DNAs of strains listed in Table 1 were prepared as described previously (9). G+C contents of DNAs were determined by the high-performance liquid chromatography method described by Kumagai et al. (10). DNA-DNA hybridization experiments were performed by using the membrane filter method described previously (2). Briefly, reference DNA was labeled with tritium by nick translation by using a nick translation kit (Takara Shuzo Co., Ltd., Kyoto, Japan). About $10 \mu \mathrm{g}$ (the exact amount used was recorded) of unlabeled single-stranded DNA immobilized on a nitrocellulose membrane and $0.1 \mu \mathrm{g}$ of labeled reference DNA were reassociated in a solution containing $0.1 \%$ sodium dodecyl sulfate and $2 \times \mathrm{SSC}(1 \times \mathrm{SSC}$ is $0.15 \mathrm{M} \mathrm{NaCl}$ plus $0.015 \mathrm{M}$ sodium citrate). After incubation for $40 \mathrm{~h}$ at $62^{\circ} \mathrm{C}$, the filters were washed and dried. Radioactivity was measured with an Aloca model LSC-3500E liquid scintillation counter. Each hybridization experiment was performed in triplicate, and the same experiment was repeated two or three times for some strains, as indicated in Table 1.

\section{RESULTS AND DISCUSSION}

The seven strains isolated from affected flounder larvae were gram-negative, facultatively anaerobic bacilli that were motile by means of a single polar flagellum (Fig. 1). The strains were oxidase positive, susceptible to vibriostatic agent $\mathrm{O} / 129(2,4-$ diamino-6,7-diisopropylpteridine), required $\mathrm{NaCl}$ to grow, and did not accumulate poly- $\beta$-hydroxybutyrate. The $\mathrm{G}+\mathrm{C}$ contents of five strains ranged from 43 to $45 \mathrm{~mol} \%$. The seven strains which we studied belonged to two genomic species as shown below. The $\mathrm{G}+\mathrm{C}$ contents of the species 1 strains were 43.4 to $44.3 \mathrm{~mol} \%$, and the $\mathrm{G}+\mathrm{C}$ content of the single species 2 strain (strain P-8706) was $45.1 \mathrm{~mol} \%$. The strains belonging to the INFL group were identified as members of the genus Vibrio on the basis of the characteristics described above.

As determined by DNA-DNA hybridization experiments, five strains belonging to the INFL group exhibited 89 to $100 \%$ relatedness to strain F-2 ${ }^{\mathrm{T}}$. However, the levels of DNA homology between strain P-8706 and the other INFL strains were only 36 to $42 \%$ despite the phenotypic similarities of these organisms. The levels of DNA relatedness between reference strains of 29 previously described Vibrio species and strain $\mathrm{F}-2^{\mathrm{T}}$ were less than $18 \%$. Strains belonging to 18 Vibrio species also 
TABLE 1. Levels of DNA relatedness of strains $\mathrm{F}-2^{\mathrm{T}}$ and P-8706 with strains of other Vibrio species and related genera

\begin{tabular}{|c|c|c|c|}
\hline \multirow[b]{2}{*}{ Strain ${ }^{a}$} & \multirow[b]{2}{*}{ Source $^{a}$} & \multicolumn{2}{|c|}{$\%$ DNA reassociation with: } \\
\hline & & $\begin{array}{l}\text { Strain } \\
\mathrm{F}-2^{\mathrm{T}}\end{array}$ & $\begin{array}{l}\text { Strain } \\
\text { P-8706 }\end{array}$ \\
\hline Vibrio ichthyoenteri $\mathrm{F}-2^{\mathrm{T}}\left(=\mathrm{IFO} 15847^{\mathrm{T}}\right)^{b}$ & Hiroshima Prefecture & 100 & $36(2)^{c}$ \\
\hline Vibrio ichthyoenteri F-1 (= IFO 15846) ${ }^{b}$ & Hiroshima Prefecture & $89(3)$ & $39(2)$ \\
\hline Vibrio ichthyoenteri $\mathrm{FH}-8804(=\mathrm{IFO} 15848)^{b}$ & Hiroshima Prefecture & $100(3)$ & $40(2)$ \\
\hline Vibrio ichthyoenteri F-9202 & Hiroshima Prefecture & $94(2)$ & $40(1)$ \\
\hline Vibrio ichthyoenteri MF-1 & Mie Prefecture & $92(3)$ & $39(2)$ \\
\hline Vibrio ichthyoenteri $\mathrm{P}-8603^{b}$ & Wakayama Prefecture & $90(3)$ & $42(2)$ \\
\hline Vibrio species INFL-2 strain P- $8706^{b}$ & Wakayama Prefecture & $37(3)$ & 100 \\
\hline Vibrio aestuarianus ATCC $35048^{\mathrm{T}}$ & ATCC & $11(2)$ & $10(1)$ \\
\hline Vibrio alginolyticus HUFP $9107^{\mathrm{T}}$ & U. Simidu & $11(2)$ & $8(1)$ \\
\hline Vibrio anguillarum HUFP $5001^{\mathrm{T}}$ & M. Nishibuchi & $14(2)$ & $11(1)$ \\
\hline Vibrio campbellii HUFP $9109^{\mathbf{T}}$ & U. Simidu & $11(2)$ & $14(1)$ \\
\hline Vibrio carchariae HUFP $9110^{\mathrm{T}}$ & U. Simidu & $11(2)$ & $12(1)$ \\
\hline Vibrio cholerae HUFP 5004 & M. Nishibuchi & $11(2)$ & $5(1)$ \\
\hline Vibrio cincinnatiensis ATCC $35912^{\mathrm{T}}$ & ATCC & $9(2)$ & \\
\hline Vibrio damsela ATCC $33539^{\mathrm{T}}$ & ATCC & $5(2)$ & $4(1)$ \\
\hline Vibrio diazotrophicus HUFP $9301^{\mathrm{T}}$ & H. Kawasaki & $11(2)$ & \\
\hline Vibrio fischeri ATCC $7744^{\mathrm{T}}$ & ATCC & $9(1)$ & \\
\hline Vibrio fluvialis HUFP $9302^{\mathrm{T}}$ & H. Kawasaki & $11(2)$ & \\
\hline Vibrio gazogenes HUFP $9303^{\mathrm{T}}$ & H. Kawasaki & $6(2)$ & \\
\hline Vibrio harveyi HUFP $9111^{\mathrm{T}}$ & U. Simidu & $12(2)$ & $13(1)$ \\
\hline Vibrio logei HUFP $5007^{\mathrm{T}}$ & M. Nishibuchi & $8(2)$ & \\
\hline Vibrio mediterranei ATCC $43341^{\mathrm{T}}$ & ATCC & $7(2)$ & $4(1)$ \\
\hline Vibrio natriegens ATCC $14048^{\mathrm{T}}$ & ATCC & $7(2)$ & $7(1)$ \\
\hline Vibrio navarrensis ATCC $51183^{\mathrm{T}}$ & ATCC & $10(2)$ & \\
\hline Vibrio nereis HUFP $9112^{\mathrm{T}}$ & U. Simidu & $15(2)$ & $13(1)$ \\
\hline Vibrio nigripulchritudo ATCC $27043^{\mathrm{T}}$ & ATCC & $6(2)$ & \\
\hline Vibrio ordalii ATCC $33509^{\mathrm{T}}$ & ATCC & $8(2)$ & $6(1)$ \\
\hline Vibrio orientalis ATCC $33934^{\mathrm{T}}$ & ATCC & $18(2)$ & $18(1)$ \\
\hline Vibrio parahaemolyticus HUFP $9114^{\mathrm{T}}$ & U. Simidu & $16(2)$ & $16(1)$ \\
\hline Vibrio pelagius HUFP $9115^{\mathrm{T}}$ & U. Simidu & $12(2)$ & $11(1)$ \\
\hline Vibrio penaeicida $\mathrm{KH}-1^{\mathrm{T}}$ & Kagoshima Prefecture & $8(1)$ & \\
\hline Vibrio proteolyticus HUFP $9307^{\mathrm{T}}$ & H. Kawasaki & $8(2)$ & \\
\hline Vibrio salmonicida ATCC $43839^{\mathrm{T}}$ & ATCC & $8(1)$ & \\
\hline Vibrio splendidus HUFP $9117^{\mathrm{T}}$ & U. Simidu & $10(2)$ & $13(1)$ \\
\hline Vibrio tubiashii HUFP $9118^{\mathrm{T}}$ & U. Simidu & $11(2)$ & $12(1)$ \\
\hline Vibrio vulnificus HUFP $5002^{\mathrm{T}}$ & M. Nishibuchi & $15(2)$ & $15(1)$ \\
\hline Photobacterium phosphoreum HUFP $9311^{\mathrm{T}}$ & H. Kawasaki & $7(1)$ & \\
\hline Escherichia coli $\mathrm{K}-12$ & IAM & $3(3)$ & $2(2)$ \\
\hline
\end{tabular}

${ }^{a}$ ATCC, American Type Culture Collection, Rockville, Md.; HUFP, Hiroshima University Fish Pathology Laboratory Culture Collection, Higashihiroshima-shi, Hiroshima, Japan; IAM, Institute of Applied Microbiology, The University of Tokyo, Tokyo, Japan; IFO, Institute for Fermentation Osaka, Osaka, Japan.

${ }^{b}$ Strains whose $\mathrm{G}+\mathrm{C}$ content was determined.

"The numbers in parentheses are the numbers of times that the experiments were performed.

exhibited low levels of DNA relatedness with strain P-8706 (less than 18\%). These results indicate that the INFL group consists of two genomic species. Species 1 contains six strains and is represented by strain $\mathrm{F}-2^{\mathrm{T}}$, and species 2 contains one

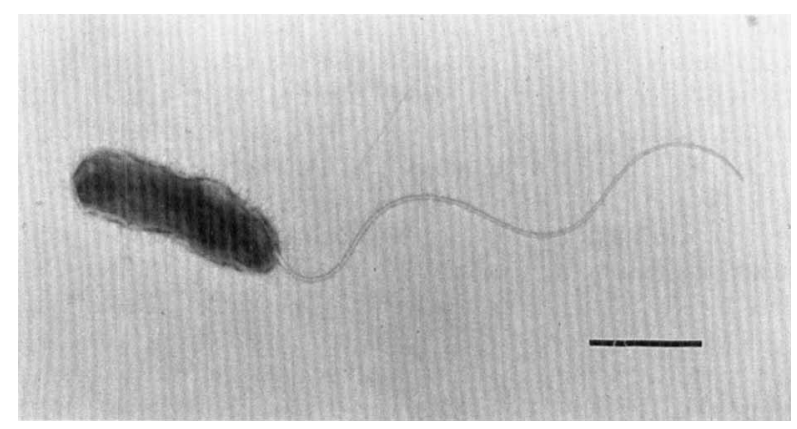

FIG. 1. Electron micrograph of a negatively stained cell of $V$, ichthyoenter $\mathrm{F}-2^{\mathrm{T}}$ showing the single polar flagellum. Bar $=1 \mu \mathrm{m}$. strain (strain P-8706). Both of these species are distinct genetically from the previously described Vibrio species examined. The following eight species were not used in the DNA-DNA hybridization experiments performed with strain $\mathrm{F}-2^{\mathrm{T}}$; Vibrio costicola, Vibrio furnissii, Vibrio hollisae, Vibrio iliopiscarius, Vibrio marinus, Vibrio metschnikovii, Vibrio mimicus, and Vibrio mytili. Three of these species, $V$. costicola, $V$. furnissii, and $V$. hollisae, differ from the flounder pathogens in their DNA G+C contents $(50 \mathrm{~mol} \%)$. These species can also be differentiated from the two species of flounder pathogens by the following phenotypic characteristics, as well as the differential characteristics shown in Table 2: V. costicola is positive in the VogesProskauer test and grows in the presence of $8 \% \mathrm{NaCl}(19) ; \mathrm{V}$. furnissii produces gas from D-glucose, grows when no $\mathrm{NaCl}$ is present, and utilizes $\gamma$-aminobutyrate and $\beta$-hydroxybutyrate (8); and $V$. hollisae produces indole and acid from L-arabinose and sucrose (7). V. iliopiscarius can be differentiated from flounder isolates by its DNA $\mathrm{G}+\mathrm{C}$ content $(40 \mathrm{~mol} \%)$ and the following characteristics: it produces arginine dihydrolase and lysine decarboxylase; it produces gas from D-glucose; it is pos- 
TABLE 2. Characteristics that distinguish $V$. ichthyoenteri from previously described Vibrio species ${ }^{a}$

\begin{tabular}{|c|c|c|c|c|c|c|c|c|c|}
\hline \multirow{2}{*}{ Taxon } & \multirow{2}{*}{$\begin{array}{c}\text { Thornley arginine } \\
\text { dihydrolase } \\
\text { activity }\end{array}$} & \multirow{2}{*}{$\begin{array}{l}\text { Nitrate } \\
\text { reduction }\end{array}$} & \multirow{2}{*}{$\begin{array}{l}\text { Chitinase } \\
\text { activity }\end{array}$} & \multirow{2}{*}{$\begin{array}{c}\text { Gelatinase } \\
\text { activity }\end{array}$} & \multirow{2}{*}{$\begin{array}{l}\text { Lipase } \\
\text { activity }\end{array}$} & \multicolumn{2}{|c|}{ Utilization of: } & \multirow{2}{*}{$\begin{array}{l}\text { Growth at } \\
35^{\circ} \mathrm{C}\end{array}$} & \multirow{2}{*}{$\begin{array}{l}\text { 3-12 polar } \\
\text { flagella }\end{array}$} \\
\hline & & & & & & D-Cellobiose & Citrate & & \\
\hline Vibrio ichthyoenteri & $-{ }^{c}$ & + & - & - & - & - & - & - & - \\
\hline Vibrio species INFL-2 & - & + & - & - & - & - & - & - & - \\
\hline Vibrio aestuarianus & + & ND & ND & - & ND & ND & + & + & - \\
\hline Vibrio alginolyticus & - & + & + & + & + & - & + & + & - \\
\hline Vibrio anguillarum & + & + & + & + & + & + & + & + & - \\
\hline Vibrio campbellii & - & + & + & + & + & $\mathrm{V}$ & $\mathrm{V}$ & + & - \\
\hline Vibrio carchariae ${ }^{d}$ & - & + & + & + & + & ND & - & + & - \\
\hline Vibrio cholerae & - & + & + & + & + & - & V & + & - \\
\hline Vibrio cincinnatiensis & - & + & + & - & - & + & + & + & - \\
\hline Vibrio costicola & $\mathrm{V}$ & $\mathrm{V}$ & - & - & - & - & - & + & - \\
\hline Vibrio damsela & + & + & + & - & - & - & - & + & ND \\
\hline Vibrio diazotrophicus & + & + & - & - & - & + & + & + & - \\
\hline Vibrio fischeri & - & V & V & - & + & + & V & V & + \\
\hline Vibrio fluvialis & + & + & + & + & + & V & + & + & - \\
\hline Vibrio furnissii & + & + & + & + & V & - & + & + & ND \\
\hline Vibrio gazogenes & - & - & - & + & + & + & + & + & - \\
\hline Vibrio harveyi & - & + & + & + & + & + & + & + & - \\
\hline Vibrio hollisae & - & + & ND & - & - & ND & - & + & - \\
\hline Vibrio iliopiscarius ${ }^{e}$ & + & + & ND & - & ND & - & - & - & ND \\
\hline Vibrio logei & - & + & + & - & $\mathrm{V}$ & + & - & - & + \\
\hline Vibrio marinus & - & + & + & + & + & - & - & - & - \\
\hline Vibrio mediterranei & + & + & - & - & + & + & ND & ND & - \\
\hline Vibrio metschnikovii & + & - & + & + & + & - & $\mathrm{V}$ & + & - \\
\hline Vibrio mimicus & - & + & ND & ND & - & ND & + & + & - \\
\hline Vibrio mytilif & + & + & ND & - & + & + & $\mathrm{V}$ & + & - \\
\hline Vibrio natriegens & - & + & - & V & + & V & + & + & - \\
\hline Vibrio navarrensisg & - & + & ND & + & ND & $\mathrm{V}$ & V & + & - \\
\hline Vibrio nereis & + & + & $\mathrm{V}$ & $\mathrm{V}$ & - & - & + & + & - \\
\hline Vibrio nigripulchritudo & - & + & + & + & + & + & + & - & - \\
\hline Vibrio ordalii & - & - & V & + & - & - & + & - & - \\
\hline Vibrio orientalis & V & + & + & + & + & + & + & + & - \\
\hline Vibrio parahaemolyticus & - & + & + & + & + & - & + & + & - \\
\hline Vibrio pelagius biovar 1 & - & + & $\mathrm{V}$ & - & + & - & + & + & - \\
\hline Vibrio pelagius biovar 2 & - & + & + & + & + & - & + & + & - \\
\hline Vibrio penaeicida & - & + & + & + & + & $\mathrm{V}$ & V & - & - \\
\hline Vibrio proteolyticus & + & + & + & + & + & - & + & + & - \\
\hline Vibrio salmonicida & - & - & - & - & - & - & ND & - & + \\
\hline Vibrio splendidus biovar 1 & V & + & + & + & + & + & + & $\mathrm{V}$ & - \\
\hline Vibrio splendidus biovar 2 & V & + & + & + & + & $\mathrm{V}$ & + & - & - \\
\hline Vibrio tubiashii & + & + & + & + & + & + & + & $\mathrm{V}$ & - \\
\hline Vibrio vulnificus biovar 1 & - & + & + & + & + & + & + & + & - \\
\hline Vibrio vulnificus biovar $2^{i}$ & - & + & ND & + & + & ND & + & + & - \\
\hline
\end{tabular}

${ }^{a}$ Data from reference 8 except as noted below.

${ }^{b}$ Data from reference 5 .

$c+$, positive in at least $90 \%$ of the strains; - , negative in at least $90 \%$ of the strains; $V$, variable among strains; ND, no data available.

${ }^{d}$ Data from reference 6.

- Data from reference 15 .

${ }^{f}$ Data from reference 16.

$g$ Data from reference 18 .

${ }^{n}$ Data from reference 9.

'Data from reference 17.

itive in the Voges-Proskauer test; and it grows at $4^{\circ} \mathrm{C}$ (15). It has been reported previously that the type strain of $V$. cholerae and the type strain of $V$. mimicus (strain ATCC 33653) are closely related as determined by DNA-DNA relatedness experiments (level of relatedness, 67\%) (4). However, strains $\mathrm{F}-2^{\mathrm{T}}$ and $\mathrm{P}-8706$, the representative strains of species 1 and 2 , respectively, exhibited only 11 and 5\% DNA relatedness to $V$. cholerae HUFP 5004, respectively. Therefore, the flounder pathogens (both species 1 and species 2) are genetically distinct from $V$. mimicus. The remaining three species $(V$. marinus, $V$. metschnikovii, and $V$. mytili) can be clearly distinguished from the flounder isolates by at least three characteristics, as shown in Table 2. In addition to the differences shown in Table
2, the following characteristics differentiate the flounder isolates from these three species: $V$. marinus is lysine decarboxylase positive and grows at $4^{\circ} \mathrm{C}$ but not $30^{\circ} \mathrm{C}(8,19) ; V$. metschnikovii is Voges-Proskauer positive and produces amylase but not oxidase (8); and $V$. mytili produces gas from Dglucose and acid from L-arabinose but not D-mannose, utilizes $\mathrm{D}$-xylose, and grows in the presence of $8 \% \mathrm{NaCl}(16)$.

The two genomic species that make up the INFL group, species 1 and 2, can be differentiated phenotypically from each other by three characteristics, acid production from D-galactose and utilization of D-glucuronate and $\beta$-hydroxybutyrate (Table 3), and are distinct from the previously described Vibrio species (Table 2). On the basis of the results described above, 
TABLE 3. Biochemical and physiological characteristics of six $V$. ichthyoenteri strains and Vibrio species INFL-2 strain P-8706 ${ }^{a}$

\begin{tabular}{|c|c|c|c|}
\hline \multirow[b]{2}{*}{ Characteristic } & \multicolumn{2}{|c|}{ V. ichthyoenteri } & \multirow{2}{*}{$\begin{array}{l}\text { Reaction of Vibrio species } \\
\text { INFL-2 strain P-8706 }\end{array}$} \\
\hline & $\begin{array}{l}\% \text { of strains } \\
\text { positive }\end{array}$ & $\begin{array}{l}\text { Reaction of } \\
\text { strain F-2 }\end{array}$ & \\
\hline Fermentation of glucose (4 days) & 100 & $t^{b}$ & + \\
\hline Gas produced from glucose (4 days) & 0 & - & - \\
\hline Oxidase activity & 100 & + & + \\
\hline Catalase activity & 100 & + & + \\
\hline Indole production (5 days) & 0 & - & - \\
\hline Nitrate reduction ( 5 days) & 100 & + & + \\
\hline Voges-Proskauer test ( 5 days) & 0 & - & - \\
\hline Hydrogen sulfide production ( 7 days) & 0 & - & - \\
\hline Arginine dihydrolase activity (Thornley) ( 7 days) & 0 & - & - \\
\hline Arginine dihydrolase activity (Møller) ( 7 days) & 0 & - & - \\
\hline Lysine decarboxylase activity (Møller) ( 7 days) & 0 & - & - \\
\hline Ornithine decarboxylase activity (Møller) (7 days) & 0 & - & - \\
\hline ONPG hydrolysis ${ }^{c}$ & 0 & - & - \\
\hline Alginase activity ( 7 days) & 0 & - & - \\
\hline Amylase activity ( 7 days) & 0 & - & - \\
\hline Chitinase activity ( 7 days) & 0 & - & - \\
\hline Gelatinase activity ( 7 days) & 0 & - & - \\
\hline Lipase activity ( 7 days) & 0 & - & - \\
\hline PHB accumulation ( 4 days $)^{d}$ & 0 & - & - \\
\hline \multicolumn{4}{|l|}{ Growth at: } \\
\hline $4^{\circ} \mathrm{C}$ & 0 & - & - \\
\hline $15^{\circ} \mathrm{C}$ & 100 & + & + \\
\hline $30^{\circ} \mathrm{C}$ & 100 & + & + \\
\hline $35^{\circ} \mathrm{C}$ & 0 & - & - \\
\hline \multicolumn{4}{|l|}{ Growth in the presence of: } \\
\hline $0 \% \mathrm{NaCl}$ & 0 & - & - \\
\hline $1 \% \mathrm{NaCl}$ & 100 & + & + \\
\hline $3 \% \mathrm{NaCl}$ & 100 & + & + \\
\hline $6 \% \mathrm{NaCl}$ & 100 & + & + \\
\hline $8 \% \mathrm{NaCl}$ & 0 & - & - \\
\hline \multicolumn{4}{|l|}{ Acid production ( 7 days) from: } \\
\hline Adonitol & 0 & - & - \\
\hline L-Arabinose & 0 & - & - \\
\hline D-Cellobiose & 0 & - & - \\
\hline Dextrin & 0 & - & - \\
\hline Dulcitol & 0 & - & - \\
\hline Erythritol & 0 & - & - \\
\hline Fructose & 100 & + & + \\
\hline D-Galactose & 0 & - & + \\
\hline D-Glucose & 100 & + & + \\
\hline Glycerol & 0 & - & - \\
\hline Glycogen & 0 & - & - \\
\hline myo-Inositol & 0 & - & - \\
\hline Inulin & 0 & - & - \\
\hline Lactose & 0 & - & - \\
\hline Maltose & 100 & + & + \\
\hline D-Mannitol & 0 & - & - \\
\hline D-Mannose & 100 & + & + \\
\hline Melibiose & 0 & - & - \\
\hline Raffinose & 0 & - & - \\
\hline L-Rhamnose & 0 & - & - \\
\hline Salicin & 0 & - & - \\
\hline D-Sorbitol & 0 & - & - \\
\hline Sucrose & 100 & + & + \\
\hline Trehalose & 100 & + & + \\
\hline D-Xylose & 0 & - & - \\
\hline \multicolumn{4}{|c|}{ Utilization of the following compounds as sole carbon sources ( 14 days): } \\
\hline$\gamma$-Aminobutyrate & 0 & - & - \\
\hline D-Cellobiose & 0 & - & - \\
\hline Citrate (Simmons) & 0 & - & - \\
\hline L-Citrulline & 0 & - & - \\
\hline Ethanol & 0 & - & - \\
\hline D-Gluconate & 50 & - & - \\
\hline D-Glucuronate & 0 & - & + \\
\hline$\beta$-Hydroxybutyrate & 0 & - & + \\
\hline
\end{tabular}


TABLE 3-Continued

\begin{tabular}{|c|c|c|c|c|}
\hline & \multirow[b]{2}{*}{ Characteristic } & \multicolumn{2}{|c|}{ V. ichthyoenteri } & \multirow{2}{*}{$\begin{array}{l}\text { Reaction of Vibrio species } \\
\text { INFL-2 strain P-8706 }\end{array}$} \\
\hline & & $\begin{array}{l}\text { \% of strains } \\
\text { positive }\end{array}$ & $\begin{array}{l}\text { Reaction of } \\
\text { strain F-2 }\end{array}$ & \\
\hline L-Leucine & & 0 & - & - \\
\hline D-Mannitol & & 33 & - & - \\
\hline Putrescine & & 0 & - & - \\
\hline Sucrose & & 17 & - & - \\
\hline D-Xylose & & 0 & - & - \\
\hline
\end{tabular}

" All tests except the growth temperature tests were performed at $25^{\circ} \mathrm{C}$.

${ }^{b}+$, positive; - , negative.

c ONPG, $o$-nitrophenyl- $\beta$-D-galactopyranoside.

¿PHB, poly- $\beta$-hydroxybutyrate.

we propose the name Vibrio ichthyoenteri sp. nov. for the species 1 strains, including strain $\mathrm{F}-2^{\mathrm{T}}$. Species 2 , which consists of one strain, strain P-8706, is also thought to be a new species of the genus Vibrio, but we do not propose a name for this species at this time because only one strain is available and the phenotypic variability of the species is not known. We designated this second genomic species Vibrio species INFL-2.

Description of Vibrio ichthyoenteri sp. nov, Vibrio ichthyoenteri (ich.thy.o.en'te.ri. Gr. n. ichthys, fish; Gr. n. enteron, gut; Gr. gen. n. ichthyoenteri, of fish gut) cells are gram-negative short rods ( 1.6 to 2.5 by 0.6 to $0.8 \mu \mathrm{m}$ ) that are straight or slightly curved and motile by means of a single polar flagellum when the cells are grown on solid media. No lateral flagella are observed on solid media, and there is no swarming. Spores are not formed. Facultatively anaerobic. Susceptible to 2,4-diamino-6,7-diisopropylpteridine (vibriostatic agent $\mathrm{O} / 129$ ) at a concentration of $10 \mu \mathrm{g}$ per disc. Neither pigmentation nor luminescence is observed. Colonies of most strains on thiosulfate-citrate-bile-sucrose agar are yellow; strain $\mathrm{F}-2^{\mathrm{T}}$ colonies are green because of weak acid production from sucrose. Biochemical and physiological characteristics are shown in Table 3 .

The $\mathrm{G}+\mathrm{C}$ contents of the DNAs of the four strains examined range from 43.4 to $44.3 \mathrm{~mol} \%$.

$V$. ichthyoenteri is the causative agent of intestinal necrosis or bacterial enteritis of Japanese flounder larvae in Japan (14). Isolated from affected fish in hatcheries, but the distribution of this organism in the environment is not known.

The type strain is strain F-2 (= IFO 15847).

\section{ACKNOWLEDGMENTS}

We thank Usio Simidu (Hiroshima University), Hiroko Kawasaki (Institute of Applied Microbiology, The University of Tokyo), Teruo Miyazaki (Mie University), and Osamu Murata (Kinki University) for kindly supplying bacterial strains.

\section{REFERENCES}

1. Aaronson, S. 1970. Experimental microbial ecology, p. 70. Academic Press, New York.

2. Akagawa-Matsushita, M., M. Matsuo, Y. Koga, and K. Yamasato. 1992. Alteromonas atlantica sp. nov. and Alteromonas carrageenovora sp. nov., bacteria that decompose algal polysaccharides. Int. J. Syst. Bacteriol. 42:621627.
3. Citarella, R. V., and R. R. Colwell, 1970. Polyphasic taxonomy of the genus Vibrio: polynucleotide sequence relationships among selected Vibrio species. J. Bacteriol. 104:434-442.

4. Davis, B. R., G. R. Fanning, J. M. Madden, A. G. Steigerwalt, H. B. Bradford, Jr., H. L. Smith, Jr., and D. J. Brenner. 1981. Characterization of biochemically atypical Vibrio cholerae strains and designation of a new pathogenic species, Vibrio mimicus. J. Clin. Microbiol. 14:631-639.

5. Farmer, J. J., III, and F. W. Hickman-Brenner. 1992. The genera Vibrio and Photobacterium, p. 2952-3011. In A. Balows, H. G. Trüper, M. Dworkin, W. Harder, and K.-H. Schleifer (ed.), The prokaryotes, 2nd ed. Springer-Verlag, New York.

6. Grimes, D. J., J. Stemmler, H. Hada, E. B. May, D. Maneval, F. M. Hetrick, R. T. Jones, M. Stoskopf, and R. R. Colwell. 1984. Vibrio species associated with mortality of sharks held in captivity. Microb. Ecol. 10:271-282.

7. Hickman, F. W., J. J. Farmer III, D. G. Hollis, G. R. Fanning, A. G. Steigerwalt, R. E. Weaver, and D. J. Brenner. 1982. Identification of Vibrio hollisae sp. nov. from patients with diarrhea. J. Clin. Microbiol. 15:395-401.

8. Holt, J. G., N. R. Krieg, P. H. A. Sneath, J. T. Staley, and S. T. Williams (ed.). 1994. Bergey's manual of determinative bacteriology, 9th ed., p. 192-194 and 260-274. The Williams \& Wilkins Co., Baltimore.

9. Ishimaru, K., M. Akagawa-Matsushita, and K. Muroga. 1995. Vibrio penaeicida sp. nov., a pathogen of kuruma prawns (Penaeus japonicus). Int. J. Syst. Bacteriol. 45:134-138.

10. Kumagai, M., M. Fujimoto, and A. Kuninaka. 1988. Determination of base composition of DNA by high performance liquid chromatography of its nuclease P1 hydrolysate. Nucleic Acids Symp. Ser. 19:65-68.

11. Masumura, K., H. Yasunobu, N. Okada, and K. Muroga. 1989. Isolation of a Vibrio sp., the causative bacterium of intestinal necrosis of Japanese flounder larvae. Fish Pathol. 24:135-141. (In Japanese.)

12. Murata, O. 1987. An infectious disease of larval flounder showing opaque intestine. Fish Pathol. 22:59-61. (In Japanese.)

13. Muroga, K. 1992. Hatchery diseases of marine fish in Japan, p. 215-222. In M. Shariff, R. P. Subasinghe, and J. R. Arthur (ed.), Diseases in Asian aquaculture I. Fish Health Section, Asian Fisheries Society, Manila, The Philippines.

14. Muroga, K., H. Yasunobu, N. Okada, and K. Masumura. 1990. Bacterial enteritis of cultured flounder Paralichthys olivaceus larvae. Dis. Aquat. Org. 9:121-125.

15. Onarheim, A. M., R. Wiik, J. Burghardt, and E. Stackebrandt. 1994. Characterization and identification of two Vibrio species indigenous to the intestine of fish in cold sea water; description of Vibrio iliopiscarius sp. nov. Syst. Appl. Microbiol. 17:370-379.

16. Pujalte, M. J., M. Ortigosa, M. C. Urdaci, E. Garay, and P. A. D. Grimont. 1993. Vibrio mytili sp. nov., from mussels. Int. J. Syst. Bacteriol. 43:358-362.

17. Tison, D. L., M. Nishibuchi, J. D. Greenwood, and R. J. Seidler. 1982. Vibrio vulnificus biogroup 2: new biogroup pathogenic for eels. Appl. Environ. Microbiol. 44:640-646.

18. Urdaci, M. C., M. Marchand, E. Ageron, J. M. Arcos, B. Sesma, and P. A. D. Grimont. 1991. Vibrio navarensis sp. nov., a species from sewage. Int. J. Syst. Bacteriol. 41:290-294.

19. West, P. A., and R. R. Colwell. 1984. Identification and classification of Vibrionaceae-an overview, p. 285-363. In R. R. Colwell (ed.), Vibrios in the environment. John Wiley \& Sons, Inc., New York. 